\title{
Alteration of Salivary Calcium And Statherin Levels in Patients with Gingivitis And Periodontitis
}

\author{
"Ameta Primasari, ${ }^{2}$ Yumi Lindawati, ${ }^{3}$ Irma Ervina \\ Faculty Of Dentistry, University Of Sumatera Utara Alumni Street No. 2, USU's Campus, Medan \\ *Corresponding author: *Ameta Primasari
}

\begin{abstract}
Salivary proteins have an important influence in tooth enamel remineralize associated with calcium hydroxyapatite. Statherin is the most powerful protein that binds calcium hydroxyapatite in saliva than other calcium-binding protein. Infection in gingiva and periodontal caused alteration of statherin and calcium levels. Therefore, researchers wanted to determine and to compare salivary statherin and calcium levels in gingivitis and periodontitic mainly on the Indonesian as an initial data to explore the influence of statherin and calcium in patients with gingivitis and periodontitic. By spitting method, 54 samples of whole saliva taken from gingivitis and periodontitic patients ; aged 20-50 years, doesn't have antibiotic treatment in 3 months, no smoking, no areca chewing, not pregnant, lactating or menstruating. Statherin level measurement using ELISA method and calcium level by spectrophotometry method. Results showed mean concentration of statherin for gingivitis is $1,79 \mathrm{ug} / \mathrm{ml}$, while periodontitics is 2,73 ug / $\mathrm{ml}$. Mean concentration for calcium in gingivitis saliva is 5,31 mmol/l, and periodontitic is $0,18 \mathrm{mmol} / \mathrm{l}$. The Spearman's correlation test showed 2 different results from 2 groups of samples. Gingivitis group showed there is no correlation between calsium and statherin in saliva $(p>0,01)$, but periodontitic group showed there is significant correlation between calcium and statherin in saliva $(p<0,05)$. The result showed that, although both are the result of salivary excretion (statherin and calcium), it turns out to different infection, showed different result. These results suggest there is a different mechanism in both infection.
\end{abstract}

Keywords: calcium, gingivitis, periodontitic, statherin, saliva,

\section{Introduction}

Biomarkers found in saliva potential as diagnostics can be identified if the composition of the protein in the pain compared to healthy individuals, it will describe the specific pathologic condition (Amado et al, 2010). Statherin a carbohydrate-free phosphoprotein very strong binding to the surface of the tooth enamel (Nakagaki et al, 2010). Statherin-rich layer on the surface of the teeth also showed a high concentration of calcium in the venue. It is certainly better to guarantee a remineralization process. However, this can happen when ions in a free state, not any precipitation. The deposition of calcium and phosphate ions it will form crystals that contribute to dental plaque mineralization (Proctor et al, 2005).

Helmerhorst et al (Boston, 2010) states that the entire chain molecules statherin has the capacity to inhibit the deposition of calcium and phosphate. A very strong bond between statherin with hydroxyapatite associated with statherin $-\mathrm{N}$ terminal has a capacity of inhibiting the deposition of secondair calcium phosphate. C-terminal of statherin facilitates the attachment of bacteria that cause infection of the gingiva and periodontal. Statherin's layer on the tooth surface indicate high calcium zone which facilitates mineralization of dental enamel, but continuous precipitation produce the mineralization of dental plaque into calculus (Proctor, et al 2005).In this study, researchers wanted to get the calcium and statherin concentration data of saliva from healthy patients, gingivitis and periodontitis, which until now has never been done in Indonesia, especially the city of Medan. Researchers also want to know the correlation statherin concentration and salivary calcium in patients with periodontitis.

\section{Method}

Cross sectional observational study using 108 samples were divided into two groups: patients with gingivitis and periodontitis patient groups. Samples are patient in Periodontia Installation Dental Hospital Faculty of Dentistry, University of Sumatera Utara, Indonesia. The inclusion criteria in general are : age 20-40 years, good general health, have a habit of brushing with toothpaste, the number of remaining teeth at least 20 teeth. Gingivitis and periodontitis group diagnosis performed by a dentist or periodontist undergoing specialist training program periodontic. Exclusion criteria for the whole group were smokers, areca nut chewer, pregnant women, breastfeeding, menopause, wearing fixed orthodontic, wear fixed or removable dentures and, experienced a systemic disease that affects the salivary condition.

Samples of saliva were collected by spitting method which saliva is collected in the mouth without stimulation and spit out every 30 seconds for \pm 5 minutes, the subject of the sample was asked not to eat and 
drink (except mineral water) for \pm 30 minutes before saliva's collected, then sat in a state of calm and comfortable with his head tilted toward the front. Saliva is stored in a cooling pack to maintain the sample temperature $2-8{ }^{\circ} \mathrm{C}$ (appropriate procedural saliva sample storage) and then immediately taken to the laboratory for analysis. At least $1 \mathrm{ml}$ saliva sample.

Measurement of calcium concentration with Calcium Kit (QuantiChrom, DICA-500, USA) using a spectrophotometer with a wavelength of $612 \mathrm{~nm}$. Measurements statherin protein in saliva samples conducted by Human Statherin ELISA Kit (Cussabio, China) using ELISA. Samples were centrifuged at $4000 \mathrm{rpm}$ for 10 minutes at a temperature of $2-8^{\circ} \mathrm{C}$ to dispose of other particles, and then processed on ELISA.

\section{Research Result}

Table 1 showed no significant difference ( $p>0.05$ ) average concentration statherin in healthy people with periodontitis patients. Nonetheless, statherin concentration of saliva in periodontitis patients was higher than statherin saliva of healthy people.

Table 1. Average Concentration of Salivary Statherin and Calcium in Gingivitis and Periodontitis

\begin{tabular}{|c|c|c|c|c|}
\hline \multirow[b]{2}{*}{ Group } & \multicolumn{2}{|c|}{ Statherin $(\mu \mathrm{g} / \mathrm{ml})$} & \multicolumn{2}{|c|}{ Calsium (mmol/l) } \\
\hline & $\mathrm{X} \pm \mathrm{SD}$ & $\begin{array}{l}\text { Median } \\
(\min -\max )\end{array}$ & $\mathrm{X} \pm \mathrm{SD}$ & $\begin{array}{l}\text { Median } \\
(\min -\max )\end{array}$ \\
\hline Gingivitis & $1,99 \pm 1,31$ & $\begin{array}{l}1,67 \\
(0,28-5,64)\end{array}$ & $1,02 \pm 0,73$ & $\begin{array}{l}0,82 \\
(0,02-3,52) \\
\end{array}$ \\
\hline Periodontitis & $2,73 \pm 2,93$ & $\begin{array}{l}1,65 \\
(0,43-19,1)\end{array}$ & $0,18 \pm 0,34$ & $\begin{array}{l}0,07 \\
(0,004-2,15)\end{array}$ \\
\hline
\end{tabular}

The correlation between the concentration of salivary calcium in statherin with gingivitis and periodontitis group obtained in Table 2. In the group of gingivitis, there were no significant correlation between the concentration of calcium statherin with saliva ( $p>0.05$ ), with the direction of negative correlations indicate a trend opposite the concentration of calcium statherin with saliva, which means that when the concentration is low, the concentration of calcium statherin will be high, and vice versa. Whereas in the group of patients with periodontitis, obtained a significant correlation between the concentration of calcium statherin with saliva $(\mathrm{p}$ $<0.05$ ), with the direction of positive correlations indicate a trend in the direction of value between the concentration of calcium statherin with saliva, it means if a low concentration, the concentration of calcium statherin too low, and vice versa.

Table 2. Correlation Concentration Statherin with Calcium Concentration Saliva At Group Gingivitis and Periodontitis

\begin{tabular}{|l|c|}
\hline Group & Spearman's Test \\
\hline \multirow{3}{*}{ Gingivitis } & $\mathrm{r}=-0,158$ \\
& $\mathrm{p}=0,253$ \\
& $\mathrm{n}=54$ \\
\hline \multirow{3}{*}{ Periodontitis } & $\mathrm{r}=0,377$ \\
& $\mathrm{p}=0,005^{*}$ \\
$\mathrm{n}=54$
\end{tabular}

\section{Discussion And Conclusions}

In this study showed higher concentrations statherin in periodontitis, compared to gingivitis (Table 1). This result reinforces the research in vivo and in situ studies by Amado et al (2010) showed statherin saliva made a major contribution in the formation of pellicle which would develop into plaque. Statherin produced by the parotid and submandibular glands. As a tyrosine rich protein, statherin has a very strong attraction capability with hydroxyapatite (Helmerhorst et al, 2010). As a body reaction to maintain oral health homeostasis, the possibility of the body reacted by releasing more statherin when oral infections worse. Researchers assume greater concentration of salivary statherin prevent the precipitation of calcium in the plaque, causing better mineralization of the plaque into calculus. However, in this study, the opposite happened.

The concentration of salivary calcium in patients gingivitis is higher than periodontitis. This may be because in general, saliva saturated with calcium-phosphate salts are also still occur when saliva function as plaque fluid (Fabian et al, 2007), the infection process that runs continuously accelerate the process of deposition of calcium in plaque into calculus. So the longer duration of the infection occurs the more unsaturated bond salivary calcium absorbed into plaque. Salivary calcium concentrations associated with the rapid mineralization of dental plaque and increased severity of periodontitis (Kiss et al, 2010). From this statement it can be said that, high salivary calcium will worsen the prognosis of the course of a disease. The higher concentration of calcium in gingivitis showed an infection of the gingiva process leading to the course of the disease increased to periodontitis. Hassan and Sandook (2005) states salivary calcium concentration 
increased significantly in the group of patients who had dental calculus compared to the control group. The process of infection that occurs in periodontal tissues, although interrupt function statherin in saliva, and calcium statherin correlation in periodontitis showed the same direction (Table 2), that is, high calcium in conjunction with the high statherin saliva. In other words, statherin still serves to inhibit the precipitation of calcium with calcium binding saliva so it does not settle into the liquid plaque, but the work was not optimal, so the concentration of high statherin but unable to bind calcium more than in patients with gingivitis. In patients with gingivitis would indicate a correlation in the opposite direction, though not significantly. It shows the process of infection of the gingiva, disrupt work statherin, so that the high concentration of statherin unable to bind more calcium in saliva. Helmerhorst et al (Boston, 2010) states that the entire chain molecules statherin has the capacity to inhibit the deposition of calcium and phosphate. A very strong bond between statherin with hydroxyapatite associated with statherin $-\mathrm{N}$ terminal has a capacity of inhibiting the deposition of calcium phosphate secondary. For that we need further research on bonding $\mathrm{N}$ - statherin in patients infected with gingivitis and periodontitis.

The conclusion of this study is an infection of the gingiva and periodontal tissues interferes with work statherin bind salivary calcium, whereas the content of statherin and calcium in saliva are also going to affect the sustainability of the infection of the gingiva and periodontal tissues.

\section{Refferences}

[1]. Amado F, Lobo MJ, Domingues P, Duarte JA, Vitorino R., 2010. Salivary peptidomics. Expert Rev Proteomics ; 7 (5) : $709-21$.

[2]. Fabian TK, Fejerdy P and Csermely P., 2007. Saliva in health and disease, chemical biology of. In: Wiley encyclopedia of chemical biology. John Wiley \& sons, Inc. 1-9.

[3]. Fabian TK, Hermann P, Beck A, Fejerdy P and Fabian G, 2012. Salivary defense proteins : their network and role in innate and acquired oral immunity. Int. J. Mol. Sci ; $13: 4295-320$.

[4]. Hassan S and Al-Sandook T, 2005. Salivary calcium concentration in patients with high incidence of calculus formation. AlRafidain Dent J;5(1) : 88-90.

[5]. Helmerhorst EJ, Traboulsi G, Salih E and Oppenheim FG., 2010. Mass spectrometric identification of key proteolytic cleavage sites in statherin affecting mineral homeostasis and bacterial binding domains. J Proteome Res ; 9 (10) : 5413-21.

[6]. Kiss E, Sewon L, Gorzo I and Nagy K, 2010. Salivary calsium concentration in relation to periodontal health of female tobacco sokers : a pilot study. Quintessence International ; 41 (9) : 779-85.

[7]. Nakagaki H, Sekine S, Terao Y, Toe M, Tanaka M, Ito HO, et al, 2010. Fusobacterium nucleatum envelope protein fom A is immunogenic and binds to the salivary statherin-derived peptide. Infection and Immunity ; Mar : 1185-92.

[8]. Newman MG, Takei HH, Klokkevold PR and Carranza FA, 2012. Carranza's clinical perodontology. 11 ${ }^{\text {th }}$ Edition. Missouri, Elsevier. 239-47.

[9]. Proctor GB, Hamdan S, Carpenter GH and Wilde P., 2005. A statherin and calcium enriched layer at the air einterface of human parotid saliva. Biochem $\mathrm{J} ; 389$ : 111-6.

[10]. Sah N, More SP and Bhutani H, 2012. Estimation and comparison of salivary calcium levels in healthy subjects and patients with gingivitis and periodontitis : a cross sectional biochemical study. AOSR ; 2 (1) : 13-16.

*Ameta Primasari. "Alteration of Salivary Calcium And Statherin Levels in Patients with Gingivitis And Periodontitis." IOSR Journal of Dental and Medical Sciences (IOSR-JDMS) 16.7 (2017): 17-19. 